\title{
A NOVEL, HIGH-RESOLUTION RESONANT THERMOMETER USED FOR TEMPERATURE COMPENSATION OF A COFABRICATED PRESSURE SENSOR
}

\author{
Chia-Fang Chiang ${ }^{l}$, Andrew B. Graham ${ }^{2}$,Eldwin J. $\mathrm{Ng}^{l}$, Chae Hyuck Ahn ${ }^{l}$, \\ Gary J. O'Brien', and Thomas W. Kenny ${ }^{1}$ \\ ${ }^{1}$ Stanford University, Stanford, CA, USA and ${ }^{2}$ Robert Bosch RTC, Palo Alto, CA, USA
}

\begin{abstract}
Temperature compensation of a capacitive pressure sensor with a cofabricated, high-resolution, resonant thermometer is presented in this paper. The epi-seal encapsulated resonant thermometer yields a resolution of $10 \mathrm{mK}$ due to the ultra-clean operating environment. By co-locating such a temperature sensor with a pressure sensor and fabricating them in a shared substrate, temperature measurement accuracy can be greatly improved. With this arrangement for temperature compensation, the pressure sensor shows a 100x reduction in the temperature dependence and realizes sensing accuracy within $\pm 0.1 \%$ FSO for the tested temperature range between 15 to $65^{\circ} \mathrm{C}$.
\end{abstract}

\section{INTRODUCTION}

Temperature compensation of a sensor can be made purely with clever algorithms such as using the characteristic of the slow change of temperature to distinguish a rapid signal from a false temperature induced drift [1]. Depending on the applications, software-only compensation methods may become inadequate and on-site temperature sensing or even ovenization [2] needs to be implemented to achieve certain performance. The former can be performed using a microcontroller or a compensation circuit to subtract temperature effects. In such cases, the temperature sensing capability determines the effectiveness of the compensation.

While typical on-chip temperature sensors are limited to a few tenths of a Kelvin of measurement resolution [3, 4], recent developments have shown that encapsulated resonant thermometers can offer temperature resolution of $\pm 0.35 \mathrm{mK}$ [5]. As a result of the significant increase in temperature sensing capability $\left(\sim 10^{3} \mathrm{X}\right)$, there is an opportunity to co-locate accurate thermometers with MEMS devices and provide improved temperature compensation, leading to significantly more accurate MEMS sensors.

This work uses an encapsulated resonant thermometer to compensate for temperature variations of a capacitive pressure sensor that is fabricated in the same piece of silicon as the thermometer in a single process flow. Unlike other temperature compensation methods, where sensors are typically formed separately and signal conditioned using an adjacent ASIC die, the proximity of the sensors on the shared substrate used in our approach greatly reduces inaccuracy resulting from potential temperature gradients and thermal energy propagation lag time existing between adjacent sensor and ASIC die.

\section{DEVICE DEVELOPMENT}

\section{Resonant thermometer}

The resonant thermometer used in this work is a flexural mode silicon resonator in the shape of a double-ended tuning fork (DETF), as shown in figure 1 . The structure of the DETF resonator consists of two long thin beams mechanically connected on both ends. These beams are DC biased through an anchor electrode on one side to form a clamped-free beam configuration. The beams are electrostatically driven via the outer electrodes with an AC stimulus and capacitively sensed by the inner electrode. A transimpedance amplifier (TIA) is used as an electrical oscillation feedback drive circuit to maintain vibration of the tuning fork at its fundamental mode resonant frequency [2].

The resonant frequency of the DETF resonator varies with temperature primarily due to the temperature dependency of material properties such as the Young's Modulus [6, 7]. At a constant DC bias voltage, the linear temperature coefficient of frequency $\left(T C f_{l}\right)$ of the DETF resonator can be described as

$$
T C f_{1} \approx \frac{T C E_{1}+\alpha}{2}
$$

where $T C E_{l}$ is the linear temperature coefficient of elastic modulus and $\alpha$ is the linear thermal expansion coefficient of silicon. $T C f_{1}$ defines the sensitivity of the DETF resonant thermometer. Notice that equation (1) gives an approximate relation. A complete analytic expression should also consider the influence of electrostatic actuation and axial loading and is given in [6]. Axial loading is not applicable in our case as the DETF resonator is single anchored and cannot sustain axial loading. On the other hand, for the geometry of the DETF resonant thermometer and bias conditions used in this work, electrostatic actuation induces $\sim 1.3 \%$ increase in $T C f_{l}$ compared to the solely material determined $T C f_{l}$, using $T C E_{l}:-63.75 \mathrm{ppm} /{ }^{\circ} \mathrm{C}$ and $\alpha: 2.6 \mathrm{ppm} /{ }^{\circ} \mathrm{C}$ [7].

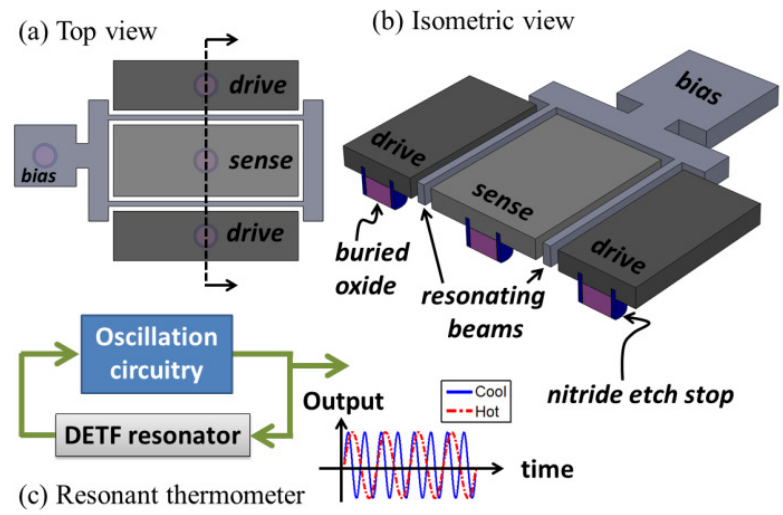

Figure 1: Schematics of a DETF resonator structure and the formation of an oscillator used as a resonant thermometer.

\section{Capacitive pressure sensor}

A capacitive absolute pressure sensor was designed to measure atmospheric pressure. Capacitive transduction was selected for its lower susceptability to temperature variations as well as the ease of fabrication integration when compared to piezoresistive transduction. Specifically, the thermal budget is not an issue for the materials used. As can be seen in figure 2, instead of using the entire diaphragm as the electrode, the device employes a reduced electrode area to increase the fractional capacitance change. This effectively improves the accuracy using our chosen measurement technique and thus can alleviate the complexity in readout circuitry design. Additionally, the temperature dependency of the sensor is reduced by sealing the capacitive cavity in low vacuum, which limits the thermal expansion effect of the trapped gas. 


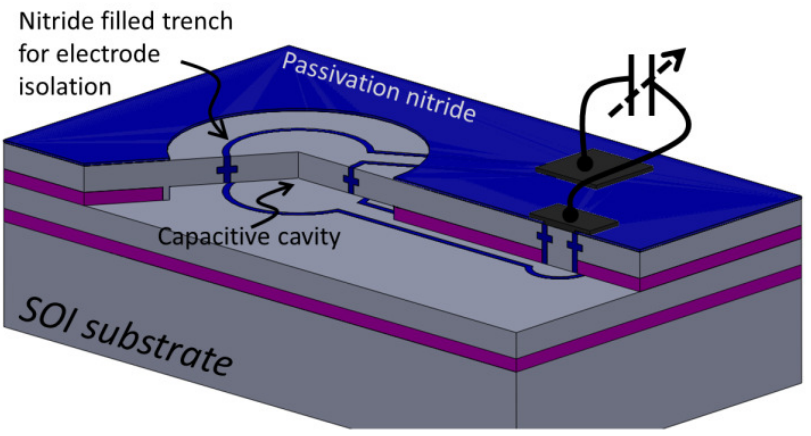

Figure 2: Structure of a capacitive pressure sensor. Electrode isolation in the diaphragm and the fixed substrate is made with a nitride filled trench.

\section{Cofabrication of the sensors}

Figure 3 shows the overall structure of the cofabricated DETF resonant thermometer and capacitive pressure sensor with their electrical functions. The integrated fabrication process is based on our previous work [8] with minor modifications. Silicon on insulator (SOI) wafers were used to construct the sensors. In the process, sacrificial silicon dioxide is used to create the cavities for the moving elements after releasing in vapor-phase hydrofluoric acid. Lateral release etch stops are created using either silicon-rich, low stress nitride or epitaxial silicon to define critical design parameters (specifically the diaphragm size of the pressure sensor), and realize a process insensitive to the release etch. After an epitaxial polysilicon deposition to seal the release etch holes (termed as "epi-seal", [9]), the deflectable diaphragm of the capacitive pressure sensor and the encapsulation of the resonant thermometer are formed simultaneously. Cross-sectional SEM images of the fabricated pressure sensor and the DETF resonant thermometer are shown in figure 4.

The epi-seal encapsulation plays a significant role in the stable performance of the DETF resonator because it provides an ultraclean, oxide-free, and hermetic environment for the resonator to operate. This frequency stability translates to high resolution of the resonant thermometer, which will be discussed in the following section.

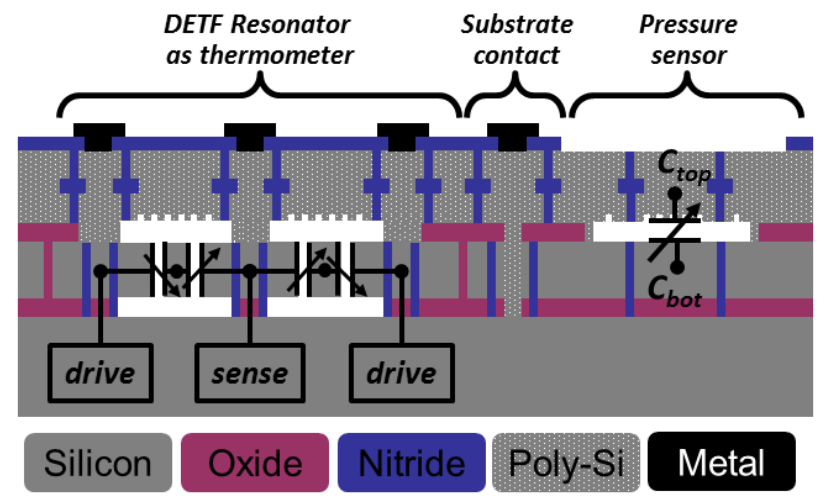

Figure 3: A cross-sectional view of the cofabricated DETF resonant thermometer and capacitive pressure sensor with their electrical functions. Structures are made with silicon on insulator wafers in a front-side only process. Electrical contacts are made with doped silicon while nitride filled trenches are used for electrical isolation.
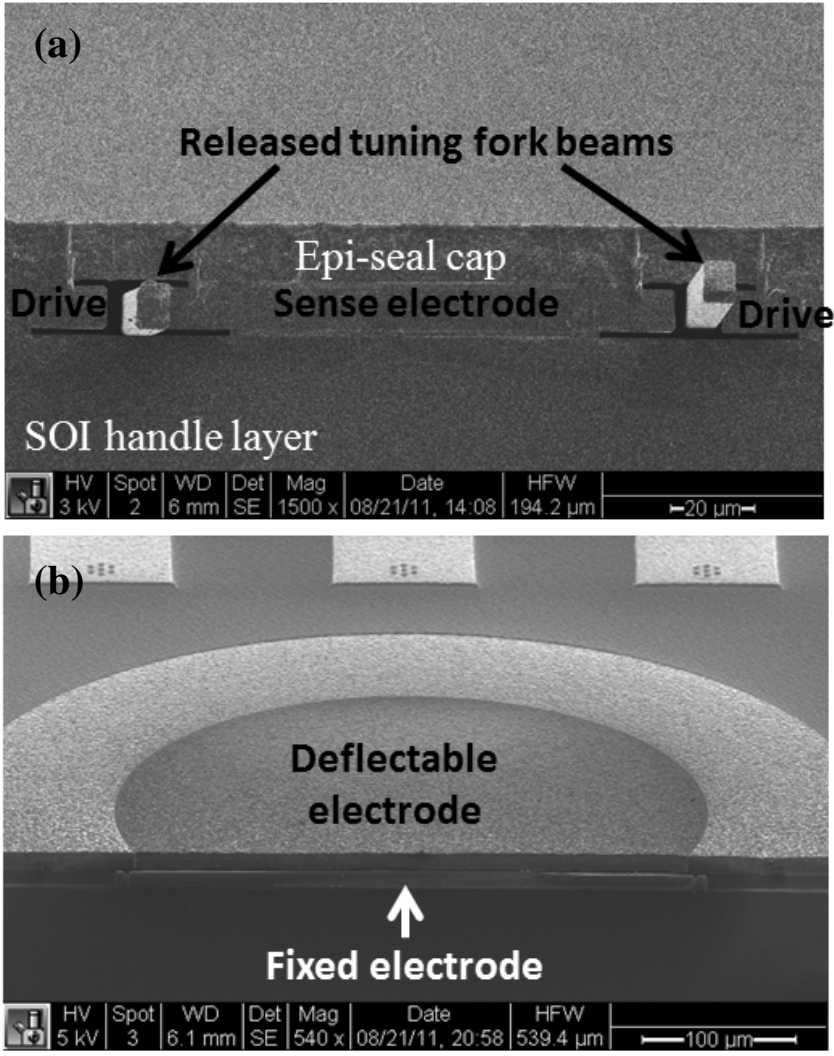

Figure 4: Cross-sectional SEM images of (a) DETF resonant thermometer and $(b)$ capacitive pressure sensor.

\section{INDIVIDUAL SENSOR PERFORMANCE}

\section{Resonant thermometer}

The sensitivity of the resonant thermometer is measured by tracking the resonant frequency change as ambient temperature varies. The test is conducted in a temperature controlled oven and the frequency is measured by using an oscillation circuit with a frequency counter. Figure 5 plots the result where the slope of $\sim$ $31 \mathrm{ppm} / \mathrm{K}$ gives the sensitivity, or $T C f_{l}$. The sensitivity could vary with silicon doping concentration and the structure orientation. Researchers have found that heavily boron doped silicon can reduce the temperature dependency and used this characteristic to demonstrate silicon resonators with highly reduced temperature sensitivity [10]. For the device fabricated in this work, the resistivity of the vibrating beams ranges from 0.22 to $0.26 \mathrm{ohm} \cdot \mathrm{cm}$. After processing, it yields sufficient conductivity for electrical access without losing temperature sensitivity.

As shown in figure 5, measurement noise causes a small error band. In order to determine the resolution of the thermometer, we used two thermometers placed side by side to track the temperature fluctuations at the same time, similar to [5]. In this test, two thermometers were placed in an oven controlled at $50 \pm 0.1{ }^{\circ} \mathrm{C}$. Thermometer 1 is the one to be calibrated and used for temperature compensation in this work. Thermometer 2 is a standard DETF resonator from a previous fabrication run, which should have very similar response to temperature, used as a reference in this case. The normalized frequency variations for the two thermometers are plotted in figures 6(a) and 6(b). By subtracting one from the other and plotting the resultant, as shown in figure 6(c), imperfections in the oven temperature control can be accounted for and thus reveal the inherent noise of the thermometer. This noise of $\sim 300 \mathrm{ppb}$ 


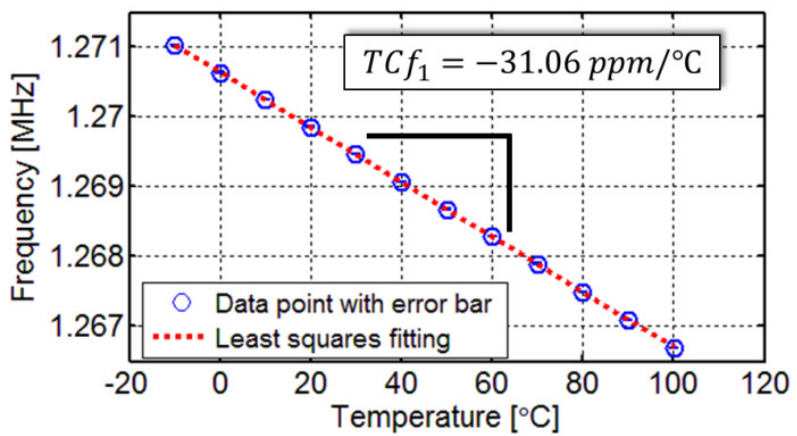

Figure 5: Measured frequency to temperature relationship of a fabricated resonant thermometer. Note the error bars are plotted and shown inside the data point circles.
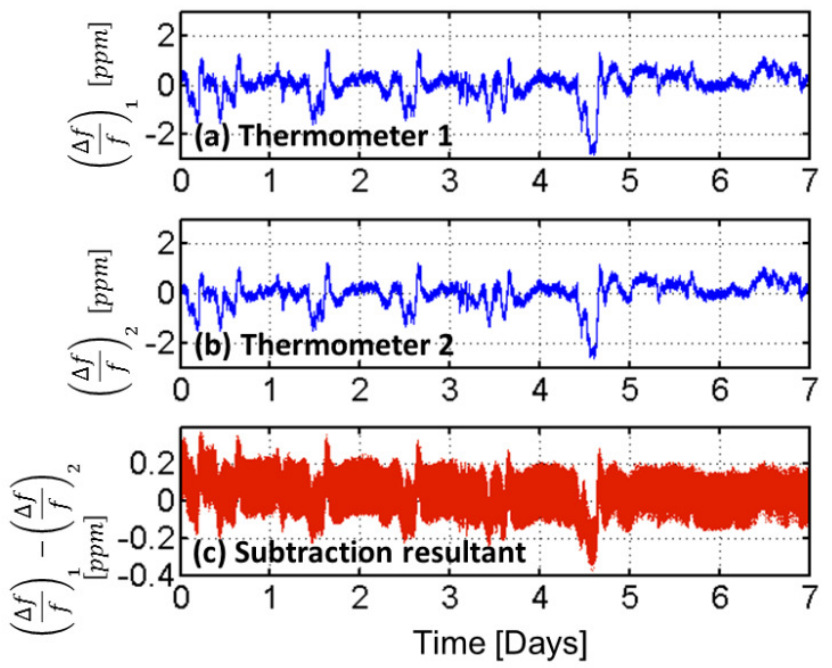

Figure 6: Stability of DETF resonant thermometers tested in a $50 \pm 0.1{ }^{\circ} \mathrm{C}$ oven. (a) and (b): frequency variations over time of two thermometers placed side by side and measured simultaneously. (c): subtraction resultant of the two resonators in order to account for the temperature control fluctuations and determine the thermometer resolution. Data is taken every second using an oscillation circuit with a frequency counter. The $\sim 300$ ppb variation corresponds to approximately $10 \mathrm{mK}$.

frequency variation is essentially the frequency stability of the DETF resonators including measurement noises from the frequency counter and oscillation circuit. Since the frequency of the resonant thermometer varies with temperature at a rate of $\sim-31$ $\mathrm{ppm} / \mathrm{K}$, the resolution of the thermometer used in the current work is approximately $10 \mathrm{mK}$. As demonstrated in [5], the measurement can be further improved by introducing the second thermometer on the same die to reduce temperature gradient effects across the two sensors.

\section{Capacitive pressure sensor}

The pressure sensor is characterized in an environmental chamber and the capacitance is readout with an Agilent 4285A LCR meter. As seen in figure 7, temperature variation causes an offset in the capacitance at a given pressure and changes the sensitivity. For the fabricated device, the capacitance offset falls in the range of 0.3 to $0.35 \mathrm{fF} /{ }^{\circ} \mathrm{C}$. This temperature dependency is undesirable and will be compensated with the co-located resonant thermometer.

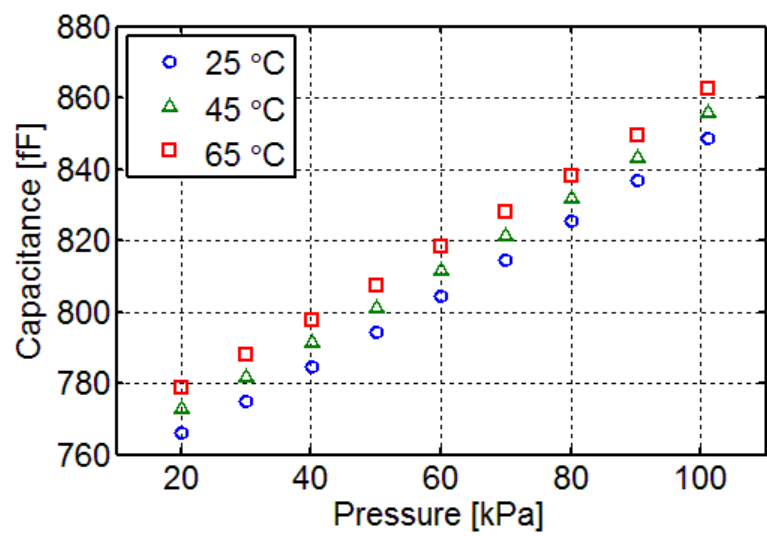

Figure 7: Capacitive response of a pressure sensor measured at various temperatures.

\section{TEMPERATURE COMPENSATION}

In order to demonstrate the feasibility of compensating for temperature induced variation of the pressure sensor output using the DETF resonant thermometer, the sensors are placed in an environmental chamber with both pressure and temperature control. The pressure is monitored by a GE Druck PACE1000 reference pressure gage with resolution of $0.0225 \mathrm{kPa}$ and adjusted by using a PFEIFFER gas dosing valve in a vacuum chamber. Temperature control is performed with a thermoelectric cooler to pump heat in and out by switching the flow direction of the actuation current.

The thermal characteristics of the pressure sensor are first captured by monitoring the capacitance at various temperatures while maintaining at constant pressure. As exemplified in figure 8, at a maintained pressure $(50 \pm 0.045 \mathrm{kPa})$, the correlation coefficient between the resonant thermometer frequency and the capacitance of the pressure sensor is found to be -0.99 over a $50 \mathrm{~K}$ temperature range. This temperature induced frequency to capacitance relationship is extracted and used for temperature compensation. A closer look at the figure, it is noticed that the capacitance exhibits a nonlinear relationship with frequency, or temperature, and a second order polynomial better fits the profile. Therefore,

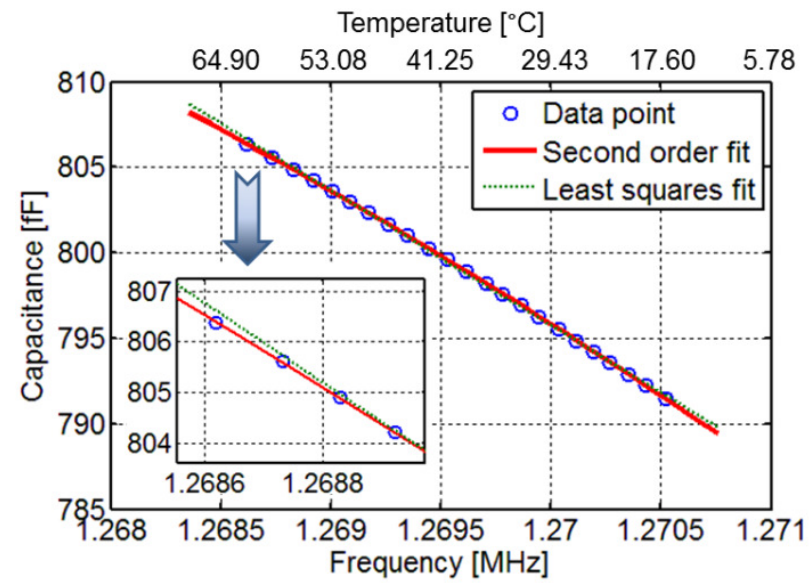

Figure 8: Correlation between the frequency of the resonant thermometer and the capacitance of the pressure sensor under a constant pressure $(50 \pm 0.045 \mathrm{kPa})$ between $15^{\circ} \mathrm{C}$ and $65^{\circ} \mathrm{C}$. A correlation coefficient of -0.99 was obtained. The inset shows a second order polynomial better fits the data. 
(a) Offset errors before and after compensation

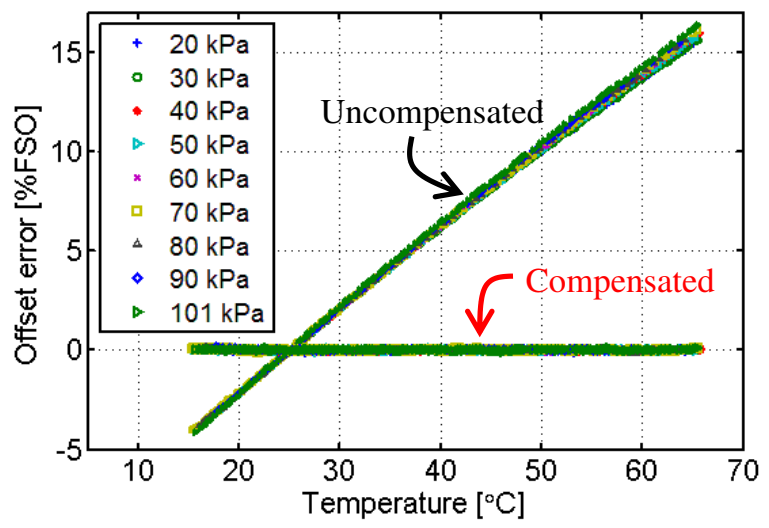

(b) Zoom in to temperature compensated data

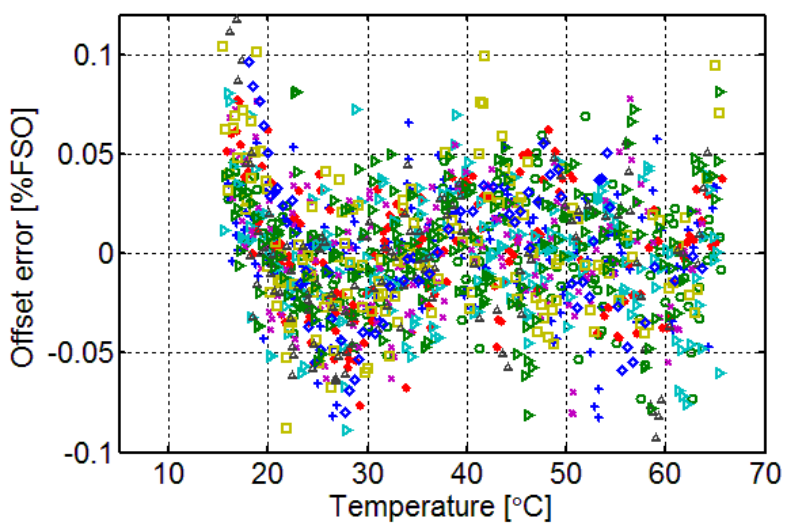

Figure 9: Offset error versus temperature ranging from 15 to 65 ${ }^{\circ} \mathrm{C}$. (a) plots data before and after temperature compensation and (b) zoom in to the offset errors of temperature compensated case. Data was compensated using the calibration result such as the one shown in figure 8 . The $\pm 0.1 \%$ FSO accuracy after compensation corresponds to $\pm 0.08 \mathrm{kPa}$.

the second order polynomial is used in temperature compensation. We performed the same characterization procedure in $10 \mathrm{kPa}$ increments from 20 to $100 \mathrm{kPa}$ to establish a complete calibration.

Figure 9 shows the resulting errors in pressure measurement as a percentage of the full scale output due to temperature variations at different pressures before and after temperature compensation. The data was normalized with respect to capacitance at $25^{\circ} \mathrm{C}$ as reference. For the temperature range investigated, the offset error before compensation is $\sim 0.5 \% \mathrm{FSO} /{ }^{\circ} \mathrm{C}$. By incorporating the calibration data from the resonant thermometer, the temperature sensitivity of the pressure sensor can be significantly reduced. Figure 9(b) enlarges the compensation result. With a few exceptions, the compensated data falls within $\pm 0.1 \%$ FSO range and is concentrated within $\pm 0.05 \%$ FSO. This $\pm 0.1 \%$ FSO variation corresponds to a resolution of $\pm 0.08 \mathrm{kPa}$. Comparing the results before and after temperature compensation, a 100 fold temperature sensitivity reduction is achieved. In addition to the pressure control errors of $\pm 0.045 \mathrm{kPa}$, the random scatter of the data distribution in figure 9(b) suggests that the temperature compensation is limited by the noise associated with the capacitance measurement. As a result, it is believed the remaining challenge to further improve the accuracy lies in the measurement setup. For example, a temperature insensitive ASIC could be mounted in the same package to convert the capacitance to voltage.

\section{CONCLUSION}

A double-ended tuning fork resonant thermometer with resolution of $10 \mathrm{mK}$ and sensitivity of $-31 \mathrm{ppm} / \mathrm{K}$ was cofabricated with a capacitive pressure sensor for temperature compensation. With the proximity of the sensors, temperature gradient and thermal lag time effects can be significantly reduced, yielding accurate temperature measurement. By using the thermometer frequency versus capacitance calibration data, the temperature dependency of the pressure sensor reduces by two orders of magnitude. With this compensation, the pressure sensor achieves measurement accuracy of $\pm 0.1 \%$ FSO in the tested range of $20-100 \mathrm{kPa}$ and $15-65^{\circ} \mathrm{C}$.

\section{ACKNOWLEDGMENTS}

The authors are grateful for the support of Robert Bosch LLC, Research and Technology Center in Palo Alto, CA. The fabrication was carried out in the Stanford Nanofabrication Facility. We would also like to thank former group members Dr. Jim Salvia and Dr. Shingo Yoneoka for the oscillation circuit design and board preparation. Travel support for Chia-Fang Chiang has been generously provided by the Transducer Research Foundation.

\section{REFERENCES}

[1] H. Weinberg, "Temperature Compensation Techniques for Low g iMEMS Accelerometers," Application Note AN-598 of Analog Devices (2002).

[2] J.C. Salvia, R. Melamud, S.A. Chandorkar, S.F. Lord and T.W. Kenny, "Real-Time Temperature Compensation of MEMS Oscillators Using an Integrated Micro-Oven and a Phase-Locked Loop," JMEMS, 19, 192 (2010).

[3] M.A.P. Pertijs, K.A.A. Makinwa, and J.H. Huijsing, “A CMOS Smart Temperature Sensor with a $3 \sigma$ Inaccuracy of $\pm 0.1{ }^{\circ} \mathrm{C}$ from $-55{ }^{\circ} \mathrm{C}$ to $125{ }^{\circ} \mathrm{C}$," IEEE Journal of Solid-State Circuits, 40, 2805 (2005).

[4] Per International Electrotechnical Commission standard, class-A Pt RTD resolution: $\pm(0.15+0.002 * \text { Temp. })^{\circ} \mathrm{C}$

[5] E.J. Ng, H.K. Lee, C.H. Ahn, R. Melamud, and T.W. Kenny, "Stability Measurements of Silicon MEMS Resonant Thermometers," Technical Digest of the 2011 Sensors Conference, Limerick, Ireland, October 28 - 31, (2011), pp. $1257-1260$.

[6] R. Melamud, "Temperature Insensitive Micromechanical Resonators," Ph.D. dissertation, Stanford Univ., CA, (2008).

[7] M.A. Hopcroft, W.D. Nix, and T.W. Kenny, "What Is The Young's Modulus of Silicon?," JMEMS, 19, 229 (2010).

[8] C.-F. Chiang, A.B. Graham, G.J. O'Brien, and T.W. Kenny, "A Single Process for Building Capacitive Pressure Sensors and Timing References with Precise Control of Released Area Using Lateral Etch Stop," Technical Digest of the 25th International Conference on Micro Electro Mechanical Systems (MEMS '12), Paris, France, January 29 - February 2, (2012), pp. $519-522$.

[9] R.N. Candler, M.A. Hopcroft, B. Kim, W.-T. Park, R. Melamud, M. Agarwal, G. Yama, A. Partridge, M. Lutz, and T.W. Kenny, "Long-Term and Accelerated Life Testing of a Novel Single-Wafer Vacuum Encapsulation of MEMS Resonators," JMEMS, 15, 1446 (2006).

[10] A.K. Samarao and F. Ayazi, "Temperature Compensation of Silicon Resonators via Degenerate Doping," IEEE Transaction on Electron Devices, 59, 87 (2012).

\section{CONTACT}

Chia-Fang Chiang, e-mail: cfchiang@mems.stanford.edu 